\title{
The merging galaxy cluster Abell 3266 at low radio frequencies
}

\author{
S. W. Duchesne, ${ }^{1,2 \star}$ M. Johnston-Hollitt, ${ }^{3}$ C. J. Riseley, ${ }^{4,5,1}$ I. Bartalucci ${ }^{6}$ and S. R. Keel ${ }^{2}$ \\ ${ }^{1}$ CSIRO Space \& Astronomy, PO Box 1130, Bentley, WA 6102, Australia \\ ${ }^{2}$ International Centre for Radio Astronomy Research (ICRAR), Curtin University, Bentley, WA 6102, Australia \\ ${ }^{3}$ Curtin Institute for Computation, Curtin University, GPO Box U1987, Perth, WA 6845, Australia \\ ${ }^{4}$ Dipartimento di Fisica e Astronomia, Università degli Studi di Bologna, via P. Gobetti 93/2, 40129 Bologna, Italy \\ ${ }^{5}$ INAF - Istituto di Radioastronomia, via P. Gobetti 101, 40129 Bologna, Italy \\ ${ }^{6}$ INAF - Istituto di Astrofisica Spaziale e Fisica Cosmica di Milano, Via A. Corti 12, 20133 Milano, Italy
}

Accepted XXX. Received YYY; in original form ZZZ

\begin{abstract}
We present new low-frequency $(v=88-216 \mathrm{MHz})$ observations of the complex merging galaxy cluster Abell 3266 . These new observations are taken with the Murchison Widefield Array (MWA) in its Phase II 'extended', long-baseline configuration, offering the highest-resolution low-frequency view of the cluster to date. We report on the detection of four steep spectrum ( $\alpha \lesssim-1$ for $S_{v} \propto v^{\alpha}$ ) extended radio sources within the cluster. We confirm the detection of a $\sim 570 \mathrm{kpc}$ radio relic to the south of the cluster, and a possible bridge of emission connecting the relic to the cluster core. We also detect two new ultrasteep-spectrum $(\alpha \lesssim-1.7)$ fossil plasma sources to the north and west of the cluster centre without associated compact radio emission. A previously detected radio galaxy in the cluster is also found to have a spectrally steepening tail with steep-spectrum components highlighted by the MWA. We do not detect a giant radio halo in the cluster. After simulating a range of radio haloes at $216 \mathrm{MHz}$ we place upper limits on the radio luminosity corresponding to $\sim 7.2 \times 10^{24} \mathrm{~W} \mathrm{~Hz}^{-1}$ at $150 \mathrm{MHz}$ assuming the expected $500 \mathrm{kpc}$ radius, up to a factor 5 lower than expected from scaling relations. Why a giant radio halo is undetected in Abell 3266 is unclear - the timeline of the merger and overall mass of the system as determined by optical and X-ray studies suggest the foundation for a hosting system.
\end{abstract}

Key words: galaxies: clusters: individual: Abell 3266 - large-scale structure of the Universe - radio continuum: general

\section{INTRODUCTION}

The observation of steep-spectrum $\left(\alpha \lesssim-1^{1}\right)$, diffuse synchtrotron radio emission in galaxy clusters provides a glimpse into the microGauss-level magnetic fields (e.g. Govoni \& Feretti 2004) and particles of the intra-cluster medium (ICM). These radio sources are thought to relate to the physical processes that shape the largest scale structures of the Universe, generated through shocks and turbulence in the ICM (for a review of diffuse radio sources in clusters, see Brunetti \& Jones 2014; van Weeren et al. 2019).

The radio sources can be broken down into a handful of distinct, but observationally similar classes. These sources include megaparsec-scale, peripherally located, shock-driven radio relics (or 'radio shocks', e.g. Johnston-Hollitt 2003; Bagchi et al. 2006; van Weeren et al. 2016; Duchesne et al. 2021a), centrally located cluster merger and turbulence-driven giant, megaparsec-scale radio haloes (e.g. Feretti et al. 2001; Wilber et al. 2020; van Weeren et al. 2021; Duchesne et al. 2021c), mini-haloes (e.g. Gitti et al. 2002; Giacintucci et al. 2014; Duchesne et al. 2021d), and a variety of other, generally smaller-scale re-accelerated/-energised radio plasmas (e.g. Slee et al. 2001; van Weeren et al. 2017; Mandal et al. 2020; Hodgson et al.

\footnotetext{
^ E-mail: stefan.duchesne.astro@gmail.com

1 We use the convention $S_{v} \propto v^{\alpha}$ for the radio spectral index, $\alpha$.
}

2021b; Duchesne et al. 2021d). Additionally, other aged - 'fossil' radio plasmas including remnant radio galaxies (e.g. Murgia et al. 2011; Duchesne et al. 2020; Quici et al. 2021) are observed in clusters. The link between these sources is not certain, and there is some suggestion of connection between the underlying particle populations and physical mechanisms as well as connections to active radio galaxies (e.g. Bonafede et al. 2014; van Weeren et al. 2017; Jones et al. 2021; Duchesne et al. 2021d). While diffuse cluster sources share similar observational properties, these sources differ fundamentally in the physical mechanisms that drive the acceleration of particles in the ICM magnetic fields even if their underlying particle populations originate from the same place. Complex merging cluster systems have been observed to host a combination of these diffuse radio sources (e.g. Pearce et al. 2017; Botteon et al. 2021; Knowles et al. 2022), with a complex variety of merger dynamics and active radio galaxies within such systems (e.g. Breuer et al. 2020).

\subsection{Abell 3266}

Abell 3266 (Abell et al. 1989) is a massive, merging galaxy cluster system located in the Horologium-Reticulum supercluster (HRS) (Fleenor et al. 2005), that contains several prominent radio-emitting objects. Over the past three decades Abell 3266 has been the subject of numerous studies particularly in the optical and X-ray regimes (e.g. 
Robertson \& Roach 1990; Quintana et al. 1996; Cypriano et al. 2001; Finoguenov et al. 2006). However, only minimal explorations have occurred at radio frequencies (Murphy 1999; Miller 2012; Dehghan 2014; Bernardi et al. 2016; Riseley 2016, and more recently by Rudnick et al. 2021), and these have been confined to frequencies above $800 \mathrm{MHz}$ leaving the cluster largely unexplored at low radio frequencies ( $\lesssim 400 \mathrm{MHz}$ ).

Finoguenov et al. (2006) noted that the cluster exhibits a low entropy region at its centre, but also that the entropy profile is significantly asymmetrical about the brightest cluster galaxy (BCG). While the low entropy region extends $\sim 8$ arcmin to the north-east, in the south-west the X-ray data show a sharp increase in entropy and temperature, bounded by a shock front (we refer the reader to fig. 1-3 in that paper). It was concluded that this was the result of a sub-cluster group in-falling from the foreground, and in the plane of the sky. However, the X-ray images alone were insufficient to prove the existence of this sub-cluster companion.

Dehghan et al. (2017) then explored the optical substructure of the system, finding five individual sub-clusters beyond the main cluster core at various stages of merging and dynamic activity. They report that the merging system is likely to have completed core passage of the main core components, and that a merger is well underway. Recently, Sanders et al. (2021) present eROSITA $^{2}$ X-ray observations of the system, further confirming the complex merger state and highlighting the numerous in-falling sub-clusters surrounding the central system.

Murphy (1999) reported re-processed observations of diffuse sources in the cluster at $843 \mathrm{MHz}$ with the Molonglo Observatory Synthesis Telescope (MOST), taken as part of the Sydney University Molonglo Sky Survey (SUMSS; Bock et al. 1999). These 843-MHz observations were also compared to higher frequency 1.4- and 2.4GHz Australia Telescope Compact Array (ATCA) data. In particular, Murphy (1999) noted the presence of a barely detectable $(2.5 \sigma)$ diffuse radio source to the south-east of the cluster in the $843-\mathrm{MHz}$ data and not present in the higher frequency images. Given the low significance of this detection, the source was thought unlikely to be real. Miller (2012) and Dehghan (2014) considered 56 hours of available 1.4-GHz ATCA data of the cluster, but did not cover the region associated with the diffuse source reported by Murphy (1999), instead focusing on other compact sources in the region.

Bernardi et al. (2016) and Riseley (2016) observed the cluster with the KAT- $7^{3}$ at $1.86 \mathrm{GHz}$ and at 1.83 and $1.32 \mathrm{GHz}$, respectively. Bernardi et al. (2016) conclude the central emission in the cluster is composed of discrete radio sources and find no evidence of peripheral or other steep-spectrum emission that was not already revealed in the SUMSS image. Riseley (2016), however, notes there may be a hint of excess central diffuse emission after subtraction of contaminating discrete sources. The low resolution of the KAT-7 observations ( $~$ 3.5 arcmin) limited the interpretation of this excess central emission.

Abell 3266 was observed as part of the MeerKAT Galaxy Cluster Legacy Survey (MGCLS; Knowles et al. 2022), where the candidate diffuse emission reported Murphy (1999) was confirmed to be a peripheral relic. In addition to this, Rudnick et al. (2021) explored the smaller-scale structure of one of the cluster radio galaxies, highlighting its complex morphology with filamentary features and evidence of episodic activity.

In this work, we present new radio images of the Abell 3266 system from the Murchison Widefield Array (MWA; Tingay et al. 2013)

\footnotetext{
2 The extended ROentgen Survey with an Imaging Telescope Array (Predehl et al. 2021).

3 Karoo Array Telescope.
}

in its Phase II 'extended' configuration (Wayth et al. 2018, hereafter, MWA-2), providing the deepest, highest-resolution low-frequency view of this cluster to date. Planck Collaboration et al. (2016) report a cluster mass of $6.64_{-0.12}^{+0.11} \times 10^{14} \mathrm{M}_{\odot}{ }^{4}$ and Dehghan et al. (2017) report a mean cluster core redshift of $z=0.0594 \pm 0.0005$. Throughout this paper, a standard $\Lambda$ Cold Dark Matter cosmology with $H_{0}=70 \mathrm{~km} \mathrm{~s}^{-1} \mathrm{Mpc}^{-1}, \Omega_{\mathrm{M}}=0.3$, and $\Omega_{\Lambda}=1-\Omega_{\mathrm{M}}$ is assumed. At the redshift of Abell $3266(z=0.0594) 1^{\prime}$ corresponds to $69 \mathrm{kpc}$.

\section{DATA}

This work is largely focused on low-frequency radio observations of Abell 3266, and makes use of data products from the MWA for characterisation of diffuse radio sources in the cluster. We supplement these data with the low-resolution $1372 \mathrm{MHz}$ KAT-7 image published by Riseley (2016, see their Chapter 3 for data reduction and imaging details), and relevant observation and image details are shown in Table 1 of this work.

\subsection{MWA}

The cluster has been observed by the MWA-2 in a 2-min snapshot mode, with observations centered on $88,118,154,185$, and $216 \mathrm{MHz}$ with a 30-MHz bandwidth each. Due to the large field-of-view (FoV) of the MWA, snapshot observations within $\sim 5$ degrees are included for imaging in this work. At the lowest frequencies, more snapshots could be included due to the increase in FoV. However, at the lower frequencies we become more limited by confusion rather than sensitivity within and near the cluster. The MWA-2 fields ' 7 ' and ' 10 ' presented in Duchesne et al. (2021d) along with supplementary data from miscellaneous projects in the region are processed and co-added. Data processing follows the procedure outlined by Duchesne et al. (2020, 2021c,d) using the MWA calibration and imaging pipeline, PIIP ${ }^{5}$, and associated software. Namely, individual 2-min snapshots are calibrated and imaged independently prior to linear co-addition/mosaicking. Calibration takes place in-field with a local sky model generated from the GaLactic and Extragalactic All-sky MWA (GLEAM) survey (Wayth et al. 2015; Hurley-Walker et al. 2017) and SUMSS (see Duchesne et al. 2020, for details) and multiscale, multi-frequency deconvolution is performed using wSCLEAN (Offringa et al. 2014; Offringa \& Smirnov 2017) with the WGRIDDER algorithm (Arras et al. 2021; Ye et al. 2021). General astrometry and brightness scaling prior to co-addition is also as detailed in Duchesne et al. (2020) and makes use of the aforementioned local sky model. This results in an overall flux scale uncertainty of $\sim 10$ per cent, predominantly inherited from the catalogues that comprise the sky model.

At $216 \mathrm{MHz}$, with the smallest FoV the contribution from the more distant field ' 7 ' observations is minimal, however, the cluster has been observed more extensively at $216 \mathrm{MHz}$ with a total integration time over all snapshots of $222 \mathrm{~min}$. The low elevation of most of the individual snapshots, particularly at $216 \mathrm{MHz}$, results in lessened sensitivity across the main lobe of the primary beam.

\footnotetext{
4 Through the strength of the Sunyaev-Zeldovich (SZ) effect, though note a number of masses have been estimated for the cluster: $M_{500}=(4.11 \pm$ $0.96) \times 10^{14} \mathrm{M}_{\odot}$ (Reichardt et al. 2013), $M_{\mathrm{X}, 500} \sim 4.6 \times 10^{14} \mathrm{M}_{\odot}$ (Piffaretti et al. 2011), $M_{500}=(8.80 \pm 0.57) \times 10^{14} \mathbf{M}_{\odot}$ (Ettori et al. 2019), through SZ, X-ray, and optical methods, respectively.

5 https://gitlab.com/Sunmish/piip
} 
Surface brightness / mJy beam ${ }^{-1}$

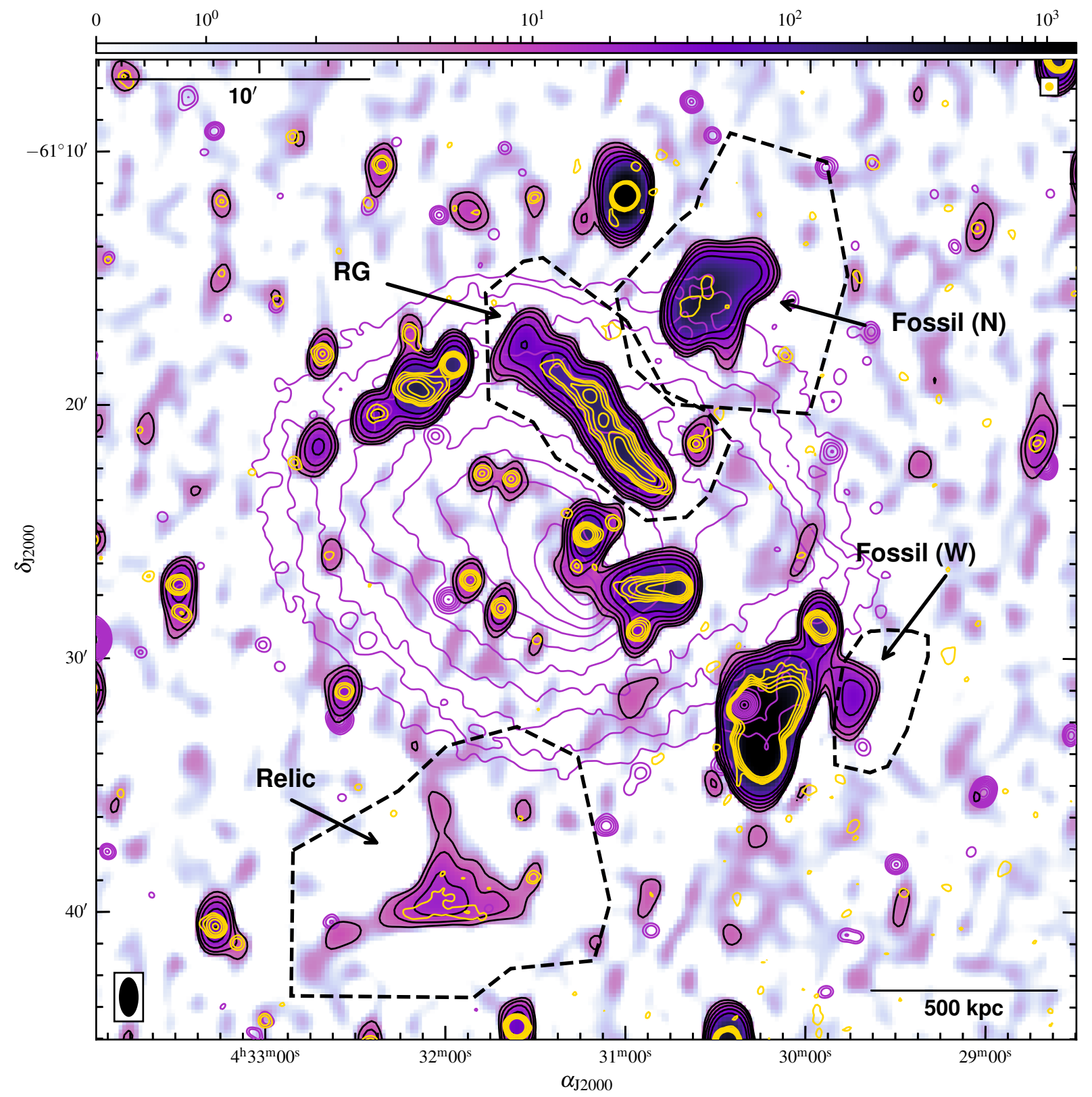

Figure 1. Abell 3266 at $216 \mathrm{MHz}$. The background image is the 216-MHz, robust $+0.5 \mathrm{MWA}-2$ image and the black contours correspond to the background image with levels of $[3,6, \ldots, 48] \times \sigma_{\mathrm{rms}}$, where $\sigma_{\mathrm{rms}}=1.3 \mathrm{mJy} \mathrm{beam}^{-1}$. The overlaid contours in yellow are the re-imaged RACS robust +0.25 image at levels of $[3,6, \ldots, 48] \times \sigma_{\text {rms }}$, where $\sigma_{\text {rms }}=0.28$ mJy beam $^{-1}$. The purple contours are from the smoothed, exposure-corrected XMM-Newton image. The sources of interest are labelled, and the black, dashed polygon regions are used for integrated flux density measurements. The framed ellipses in the lower left and top right are the shapes of restoring beams for the MWA-2 and RACS images, respectively. The linear scale is at the redshift of the cluster.

This limits the sensitivity in the final images, though we still obtain a root-mean-square (rms) noise in the stacked 216-MHz image of $\sim 1.3 \mathrm{mJy}$ beam $^{-1}$ near the cluster. All imaging details are shown in Table 1.

We also make use of the 200-MHz wideband image from the GLEAM survey to complement the MWA-2 observations. Other GLEAM bands are generally less sensitive or are too confused for useful analysis in this case.

\subsection{Other data products}

The Rapid ASKAP ${ }^{6}$ Continuum Survey (RACS; McConnell et al. 2020, project AS110; Hotan et al. 2020, SB8678) is also used in helping to identify contaminating discrete sources within the lowresolution MWA and KAT-7 data. Calibrated RACS visibilities from two adjacent observations covering the cluster are retrieved from

${ }^{6}$ Australian Square Kilometre Array Pathfinder (Hotan et al. 2021). 
Table 1. Details of images used in this work.

\begin{tabular}{|c|c|c|c|c|c|c|c|}
\hline$(\mathrm{MHz})$ & Instrument & $\begin{array}{c}\tau_{\text {effective }}{ }^{b} \\
\text { (min) }\end{array}$ & Image weighting & $\begin{array}{l}\text { Restoring beam } \\
(\operatorname{arcsec} \times \operatorname{arcsec})\end{array}$ & $\begin{array}{c}\min \left(\sigma_{\mathrm{rms}}\right)^{\mathrm{c}} \\
\left(\mathrm{mJy} \mathrm{beam}^{-1}\right)\end{array}$ & $\begin{array}{l}\max (\theta)^{\mathrm{d}} \\
(\operatorname{arcmin})\end{array}$ & $\begin{array}{c}\xi_{\text {scale }}{ }^{\mathrm{e}} \\
(\%)\end{array}$ \\
\hline 88 & MWA-2 & $45(48)$ & robust $+0.5(+2.0)$ & $197 \times 124(260 \times 159)$ & $8.3(9.7$ & $120(60)$ & $10(10)$ \\
\hline 118 & MWA-2 & $57(49)$ & robust $+0.5(+2.0)$ & $156 \times 92(195 \times 117)$ & $4.1(6.0)$ & $120(60)$ & $10(9)$ \\
\hline 154 & MWA-2 & $46(56)$ & robust $+0.5(+2.0)$ & $116 \times 70(149 \times 90)$ & $2.3(4.0)$ & $120(60)$ & $9(9)$ \\
\hline 185 & MWA-2 & $30(32)$ & robust $+0.5(+2.0)$ & $111 \times 59(123 \times 76)$ & $2.8(3.0)$ & $120(60)$ & $9(9)$ \\
\hline 216 & MWA-2 & $81(68)$ & robust $+0.5(+2.0)$ & $98 \times 51(110 \times 72)$ & $1.3(1.5)$ & $120(60)$ & $10(10)$ \\
\hline $200^{f}$ & MWA & $\sim 10$ & robust -1.0 & $149 \times 132$ & 22 & $\sim 670$ & 8 \\
\hline 887 & ASKAP & 15 & robust +0.25 & $25 \times 25^{g}$ & 0.28 & 49 & 10 \\
\hline $1372^{\mathrm{h}}$ & KAT-7 & 270 & natural & $259 \times 218$ & 2.3 & 30.31 & 10 \\
\hline
\end{tabular}

Notes. For MWA-2 data, both robust +0.5 and (robust +2.0 ) images are used.

${ }^{a}$ Central frequency.

${ }^{\mathrm{b}}$ Effective integration time. For the MWA-2 data this is the total integration time for all snapshots scaled by the stacked Stokes $I$ primary beam response at the position of Abell 3266 (i.e., less than the total integration time).

${ }^{c}$ Minimum local rms noise near the cluster.

${ }^{\mathrm{d}}$ Maximum detectable angular scale. For MWA-2 robust +2.0 images this is lower as imaging with the +2.0 weighting becomes dominated by short baseline artefacts. This does not reduce sensitivity to scales of interest here by any signicant amount.

${ }^{\mathrm{e}}$ Uncertainty in the flux density scale.

${ }^{\mathrm{f}}$ See Hurley-Walker et al. (2017) for imaging details.

${ }^{\mathrm{g}}$ Restored with a circular beam.

${ }^{\mathrm{h}}$ See Riseley (2016) for imaging details.

the CSIRO ${ }^{7}$ ASKAP Science Data Archive (CASDA; Chapman et al. 2017; Huynh et al. 2020) and imaged with wsCLEAN. We use a 'Briggs' robust +0.25 image weighting following Duchesne et al. (2021d), resulting in a $25^{\prime \prime} \times 25^{\prime \prime}$ resolution image with a local rms noise of $0.28 \mathrm{mJy}_{\text {beam }}{ }^{-1}$ near the cluster. The brightness scale of this robust +0.25 image is consistent within a few per cent of the available RACS survey image for the region of interest. Further details of the RACS image are shown in Table 1.

For a qualitative overview of the cluster morphology, we use reprocessed, archival XMM-Newton data using the EPIC ${ }^{8}$ instrument 9 (PI Aschenbach, originally presented by Sauvageot et al. 2005; Finoguenov et al. 2006). For detail about the XMM-Newton data reduction, we refer to appendix A of Bartalucci et al. (2017).

\section{RESULTS AND DISCUSSION}

\subsection{The diffuse radio sources}

Figure 1 shows the cluster at $216 \mathrm{MHz}$ with the robust +0.5 MWA-2 data along with the re-imaged RACS image overlaid as contours. The XMM-Newton image is shown as purple contours highlighting the complex morphology of the ICM of the system. These data reveal numerous radio sources, and four sources are highlighted and labelled on Figure 1, including the bright, elongated radio galaxy north of the cluster centre ('RG'), what we consider a relic towards the south ('Relic'), a fossil plasma source towards the north ('Fossil (N)'), and a smaller fossil plasma source nearby the bright, complex head-tail galaxy towards the west ('Fossil (W)').

Figure 2 shows additional 88-216 MHz MWA images of the cluster, with most features present in all images. Flux densities, $S_{v}$, for all sources are integrated within the polygon regions shown on Figure 1

\footnotetext{
7 Commonwealth Scientific and Industrial Research Organisation.

8 European Photon Imaging Camera.

9 Obs. IDs: 010526100, 0105262201, 0105260901, 0105260801, 0105262001, 0105262501, 0105260701, 0105261101, 0105262101.
}

Table 2. MWA-2, GLEAM, and KAT-7 flux density $\left(S_{v}\right)$ measurements of the four sources labelled on Figure 1, integrated within the regions shown on Figure 1.

\begin{tabular}{|c|c|c|c|}
\hline $\begin{array}{r}v \\
(\mathrm{MHz})\end{array}$ & $\begin{array}{c}S_{v} \\
(\mathrm{mJy})\end{array}$ & $\underbrace{S_{v, \text { discrete }}}_{(\mathrm{mJy})}{ }^{\mathrm{a}}$ & $\alpha$ \\
\hline \multicolumn{4}{|c|}{ Fossil (N) } \\
\hline 87.7 & $5130 \pm 620$ & - & \multirow{6}{*}{$-1.70 \pm 0.14$} \\
\hline 118.4 & $3260 \pm 310$ & - & \\
\hline 154.2 & $2140 \pm 190$ & - & \\
\hline 185.0 & $1560 \pm 140$ & - & \\
\hline 200.3 & $1110 \pm 190$ & - & \\
\hline 215.7 & $1130 \pm 120$ & - & \\
\hline \multicolumn{4}{|c|}{ RG } \\
\hline 118.4 & $2050 \pm 200$ & $30 \pm 3$ & \multirow{5}{*}{$-1.26 \pm 0.22$} \\
\hline 154.2 & $1460 \pm 140$ & $22 \pm 2$ & \\
\hline 185.0 & $1140 \pm 110$ & $19 \pm 2$ & \\
\hline 200.3 & $1030 \pm 170$ & $18 \pm 1$ & \\
\hline 215.7 & $980 \pm 110$ & $16 \pm 1$ & \\
\hline \multicolumn{4}{|c|}{ Relic } \\
\hline 87.7 & $492 \pm 69$ & $21 \pm 15$ & \multirow{7}{*}{$-1.04 \pm 0.09$} \\
\hline 118.4 & $353 \pm 48$ & $17 \pm 10$ & \\
\hline 154.2 & $221 \pm 46$ & $14 \pm 7$ & \\
\hline 185.0 & $225 \pm 62$ & $13 \pm 5$ & \\
\hline 200.3 & $211 \pm 92$ & $12 \pm 5$ & \\
\hline 215.7 & $135 \pm 72$ & $11 \pm 4$ & \\
\hline 1372.0 & $28.9 \pm 6.0$ & $3.1 \pm 0.3$ & \\
\hline \multicolumn{4}{|c|}{ Fossil (W) } \\
\hline 118.4 & $292 \pm 61$ & - & \multirow{4}{*}{$-2.4 \pm 0.4$} \\
\hline 154.2 & $179 \pm 37$ & - & \\
\hline 185.0 & $113 \pm 24$ & - & \\
\hline 215.7 & $70 \pm 15$ & - & \\
\hline
\end{tabular}

${ }^{\text {a }}$ Subtracted contribution from discrete sources detected in the RACS image, assuming $\alpha=-0.7$. 


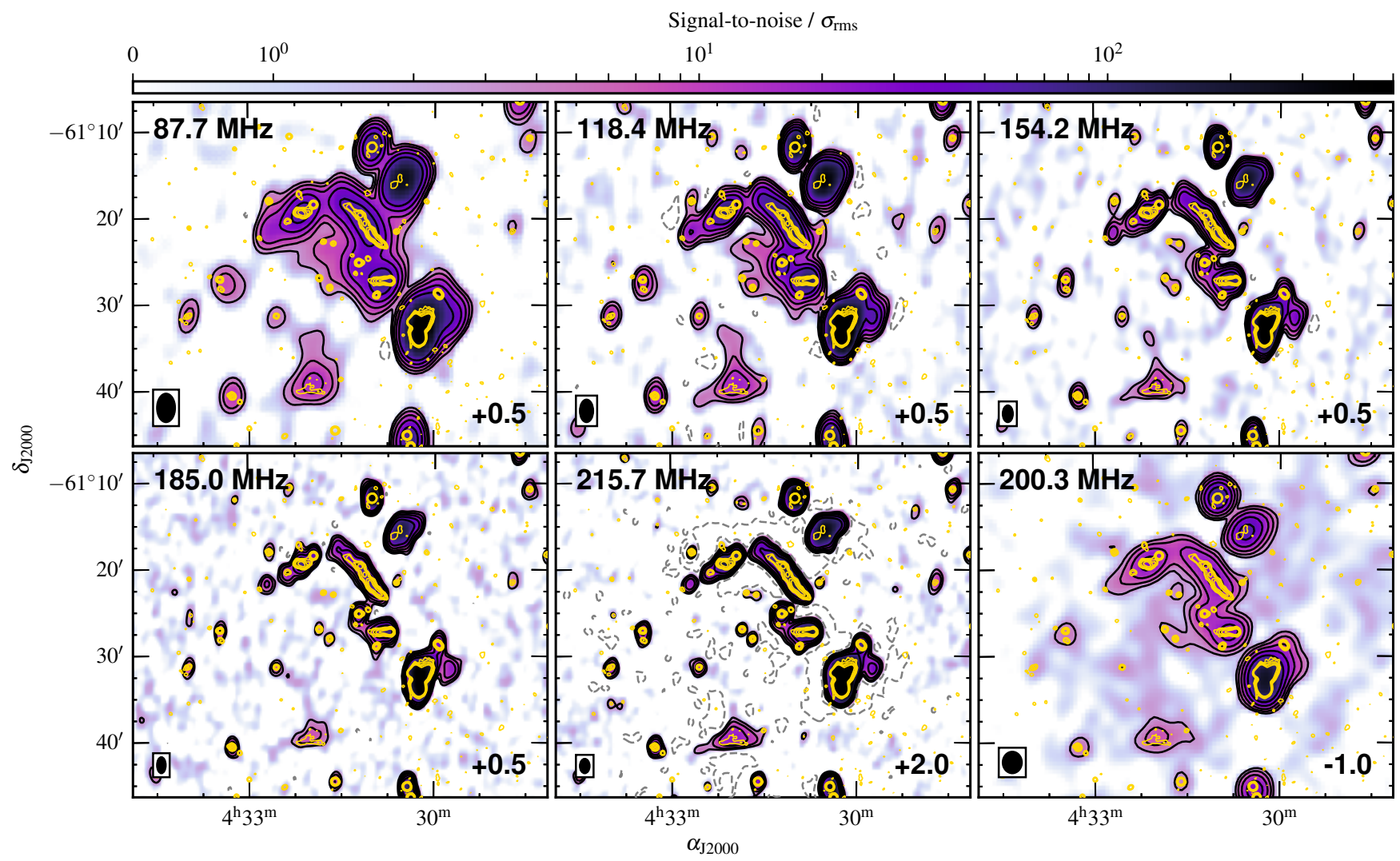

Figure 2. MWA images of Abell 3266 from 88-216 MHz. The 'Briggs' robust parameter for each image is labelled. The MWA-2, 216-MHz robust +0.5 image is shown in Figure 1 . Black contours are drawn at $[3,6,12,24,48] \times \sigma_{\text {rms }}$, with $\sigma_{\text {rms }}$ for each image reported in Table 1 , and the grey, dashed contour is set at $-3 \sigma_{\mathrm{rms}}$. Yellow contours are from the RACS image as in Figure 1. Note the colourscale for all images is mapped to $0-500 \sigma_{\mathrm{rms}}$.

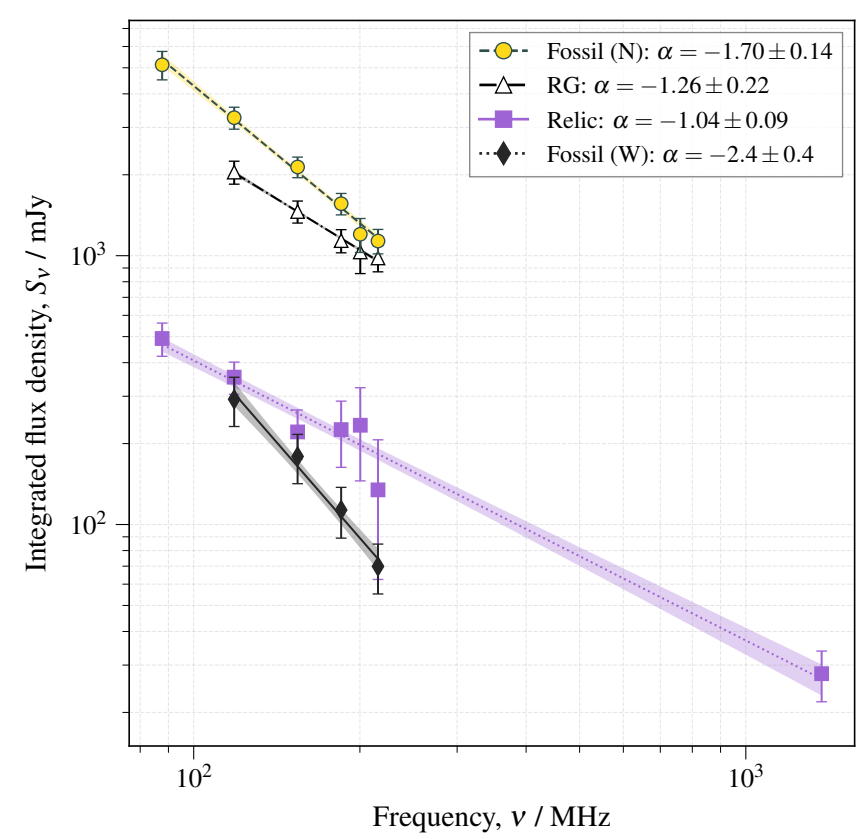

Figure 3. Integrated spectra of the four radio sources of interest. Measurements are also provided in Table 2. The sources are fit with normal power law models, and a $68 \%$ confidence interval for each fit is shown. following the process described in Section 2.4 of Duchesne et al. (2021d). Uncertainties for flux density measurements, $\sigma_{S_{v}}$, are

$\sigma_{S_{v}}{ }^{2}=N_{\text {beam }} \sigma_{\text {rms }}{ }^{2}+\left(\xi_{\text {scale }} S_{v}\right)^{2}+\sigma_{\text {discrete }}{ }^{2}$,

where $N_{\text {beam }}$ is the number of independent restoring beams covering the integration region, $\xi_{\text {scale }}$ is the flux scale uncertainty $(\sim 10$ per cent for most images), and $\sigma_{\text {discrete }}$ is the uncertainty on the flux density for subtracted discrete sources. Relevant discrete sources detected in the RACS image are subtracted by extrapolating their $887-\mathrm{MHz}$ flux density to other frequencies assuming a power law model with spectral index $\alpha=-0.7$. Additionally, $\sigma_{\text {discrete }}$ in Equation 1 incorporates both the measurement uncertainty of the discrete sources and the uncertainty in this assumed $\alpha$ by adding in quadrature the difference between the extrapolated flux densities when using a range of $\alpha$ (between -0.5 and -1.0 ; for further detail refer to Section 2.4 of Duchesne et al. 2021d). We use the robust +2.0 MWA-2 images for measuring the flux densities of all sources except the western fossil, which becomes too blended with the nearby wide-angle tail (WAT) source, PKS 0429-61. For the western fossil, we instead use the robust +0.5 MWA-2 images, convolving all maps to a common resolution (that of the $118-\mathrm{MHz}$ robust +0.5 map) to ensure any additional blending with the WAT is consistent across the band.

We note that the higher background signal in the GLEAM 200$\mathrm{MHz}$ image results in a large measurement uncertainty. Individual flux density measurements, along with subtracted discrete source contributions, are reported in Table 2. All four sources are fit with normal power law models, and the measured spectra and best-fitting models are shown on Figure 3. 


\subsubsection{Radio galaxy $(R G)$}

To the north of the cluster centre, an elongated, $630 \mathrm{kpc}$ radio galaxy is observed, detected completely across the MWA data as well as in SUMSS and the RACS image. This source is also detected in the ATCA data presented by Murphy (1999), who note a possible optical host at the south-west 'head' and a spectral steepening along the 'tail'. As seen in Figure 1, the RG is almost completely detected in the RACS image, except for the steepest-spectrum component at the 'tail' end to the NE. Observationally, the source is similar to the large headtail radio galaxy in Abell 1132 (Wilber et al. 2018). The integrated spectral index of $\alpha=-1.26 \pm 0.22$ across the MWA band highlights the contribution from the steep-spectrum components along the 'tail' of the source.

Rudnick et al. (2021) present higher-resolution MeerKAT data at $1.285 \mathrm{GHz}$ for this source, showing a complex filamentary morphology with 'ribs' and 'tethers', not discernible in the lower-resolution data presented here except as some clumping along the emission. The extent of the reported emission is similar to that seen in the RACS data. They report a faint core towards the south-west (i.e. the 'head' of the emission), associated with a spectroscopically-confirmed cluster member. Rudnick et al. (2021) show a resolved spectral index map for the source across the 900-1670 MHz MeerKAT data, highlighting this steepening from $\alpha \approx-0.6$ at the south-west 'head' of the radio emission to $\alpha \lesssim-3$ approaching the base of the north-east 'tail'. In the 216-MHz MWA-2 data shown in Figure 1, the additional component at the end of the north-east 'tail' is not detected in the high-resolution MeerKAT image.

This additional component of the tail is not measured separately in this work due to significant confusion, and a resolved spectral index map is not possible with the present MWA data. Based on the integrated spectral index we report here $(\alpha=-1.26 \pm 0.22)$ across the entire structure, observation of such steep-spectrum components undetected in the MeerKAT image implies some curvature beyond $\sim 216 \mathrm{MHz}$.

\subsubsection{Relic}

Murphy (1999) reported a $2.5 \sigma_{\text {rms }}$ detection of a candidate relic to the south-west of the cluster, which is confirmed by the $1.285-\mathrm{GHz}$ MeerKAT data published by Knowles et al. (2022). The MWA and KAT-7 data additionally detect this relic source. The (deconvolved) largest angular size is $\sim 8.3$ arcmin in the $216-\mathrm{MHz}$ image, corresponding to $\sim 570 \mathrm{kpc}$, smaller than the size reported by Knowles et al. (2022) ${ }^{10}$. The integrated spectrum between $88-1327 \mathrm{MHz}$ has a spectral index of $\alpha=-1.04 \pm 0.09$, consistent with the general relic population and as expected from diffusive-shock acceleration processes. The relic is also partially detected in the RACS image, though the emission is patchy and we note that the RACS observations have low sensitivity to angular scale approaching 10 arcmin (McConnell et al. 2020; Duchesne et al. 2021d).

As we decrease in frequency through the MWA-2 data, the relic is shown to extend further core-ward with a 'bridge' of emission extending for $\sim 4.5 \operatorname{arcmin}(\sim 310 \mathrm{kpc})$ from the relic to the cluster core. No discrete sources are detected in the RACS image at this location, and the lack of detection in the 216-MHz MWA-2 image suggests spectral steepening in this direction. Such extensions connected to radio relics have sometimes been observed to connect to

10 They report 9.7 arcmin, though after inspection of the publicly available MGCLS images we measure a largest angular extent of $\sim 8.6$ arcmin, closer to our MWA measurement. giant radio haloes, and typically show a spectral gradient steepening in the core-ward direction indicative of an ageing radio plasma (e.g. van Weeren et al. 2016).

\subsubsection{Fossil (N)}

To the north of the cluster we detect a previously unreported steepspectrum source which we consider fossil radio plasma. The source has a slightly elongated morphology with an angular size of $\sim 5.4$ arcmin ( $370 \mathrm{kpc})$ and is located in the space between the spectroscopic groups '3', '4', and '5' from Dehghan et al. (2017, see also fig. 6.6 from Dehghan 2014). No compact emission is seen in the RACS data within the emission region at $216 \mathrm{MHz}$. As with the Southern relic, the RACS image shows a patchy detection. We find $\alpha=-1.70 \pm 0.14$ across the available MWA data, and consider this another example of cluster-based fossil plasma from a long-dead active galactic nucleus (AGN).

Alternatively, the source may represent a radio phoenix or other reaccelerated fossil plasma source. The cluster is sufficiently dynamic that weak shocks will be present throughout the volume and indeed Sanders et al. (2021) report two candidate shocks, though not at this location. While not reported by Knowles et al. (2022), the source is also detected in the publicly available images from the MGCLS. The MGCLS data as well as deeper ASKAP observations will provide high frequency data points to constrain the spectrum and investigate relevant synchrotron ageing models to determine the age of the source (e.g., Riseley et al., in prep.).

\subsubsection{Fossil (W)}

The fossil source to the west of the complex WAT has an ultra-steep spectrum with $\alpha=-2.4 \pm 0.4$ between 118-216 MHz. This source is smaller than other diffuse sources in the cluster at $\sim 2.4$ arcmin $(\sim 170 \mathrm{kpc})$. It may represent a past episode from the WAT, however, it appears to be a distinct component, where the blending between the WAT and fossil is a function of image resolution rather than physical proximity. As with the northern fossil source this may also instead represent a re-accelerated plasma, though with the present data it is not possible to distinguish between a remnant or re-accelerated radio plasma.

Such ultra-steep-spectrum emission has been steadily uncovered in MWA data (Hodgson et al. 2021b; Duchesne et al. 2021d,b) and highlights a niche discovery space for the MWA and other lowfrequency inteferometers. However, with the low resolution and low signal-to-noise ratio (SNR) in these detections, uncertainties remain in the integrated spectra for these faint ultra-steep-spectrum radio sources detected by the MWA, and indeed it will not be until SKALow begins operations that we will be able to explore them in much more detail at these low frequencies.

\subsection{The absence of a giant radio halo in Abell 3266}

Many massive $\left(\gtrsim 5 \times 10^{14} \mathrm{M}_{\odot}\right)$, merging clusters have been observed to host giant ( $\gtrsim 1 \mathrm{Mpc}$ ) radio haloes (e.g. van Weeren et al. 2019, and references therein), with luminosities that have been found to scale with the cluster mass and other physical properties (e.g. Liang et al. 2000; Cassano et al. 2010; Martinez Aviles et al. 2016) at both $1.4 \mathrm{GHz}$ (e.g. Cassano et al. 2013; Duchesne et al. 2021b; Cuciti et al. 2021) and $150 \mathrm{MHz}$ (van Weeren et al. 2021; Duchesne et al. 2021c).

While Abell 3266 hosts a mix of extended, steep-spectrum radio 
Surface brightness / $\mathrm{mJy}_{\text {beam }}{ }^{-1}$

0

$10^{1}$

$10^{2}$
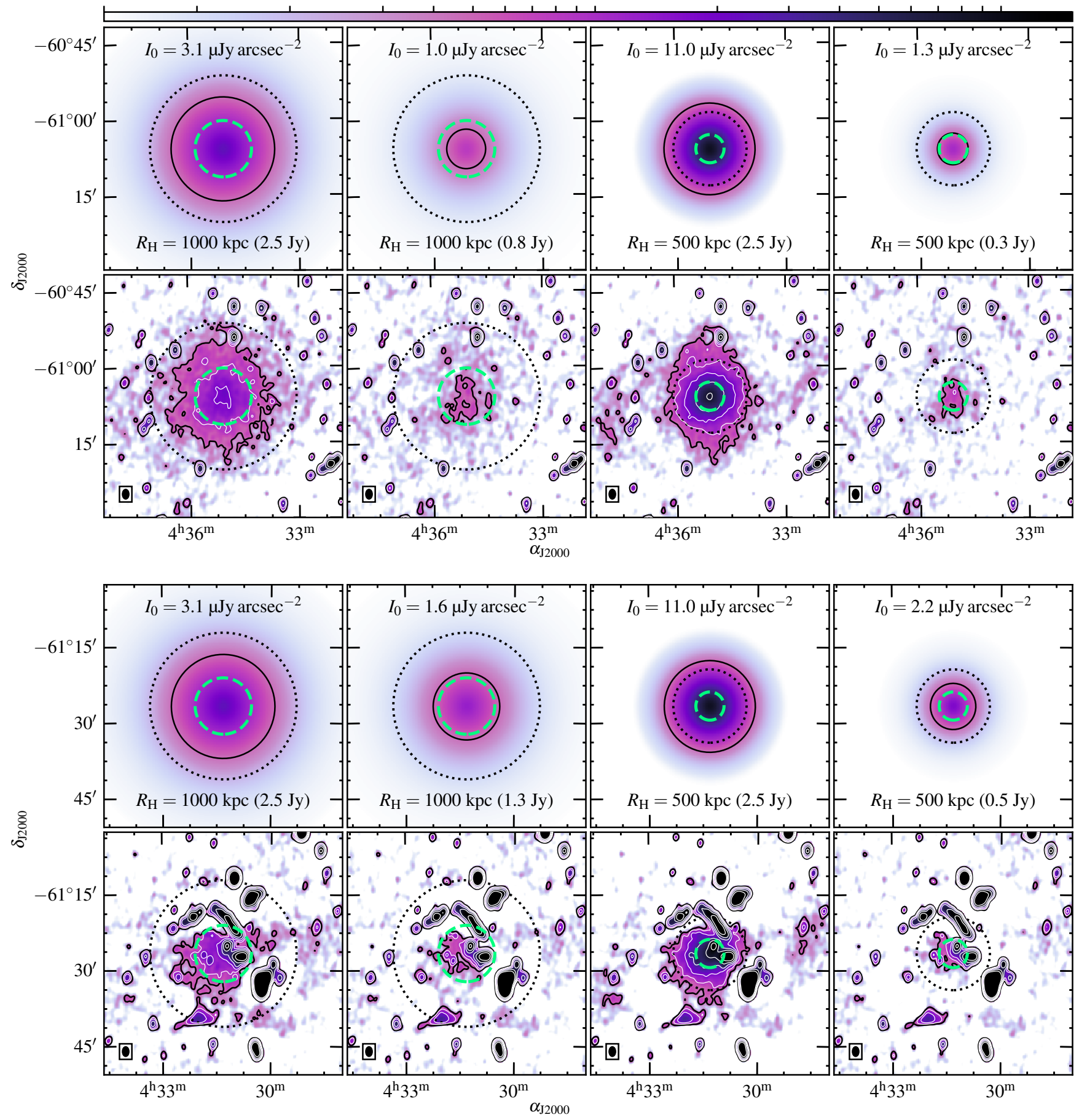

Figure 4. Mock radio haloes injected into a quiet patch of sky near Abell 3266 (top two rows) and at the cluster centre(bottom two rows). The model radio haloes are convolved with a $100^{\prime \prime} \times 70^{\prime \prime}$ beam for comparison and are shown prior to imaging and injection into the datasets. The quoted surface brightness in each panel is the peak value at $216 \mathrm{MHz}$ after injecting into the datasets. The solid contour is set at $3 \sigma_{\mathrm{rms}}\left(\sigma_{\mathrm{rms}}=1.5 \mathrm{mJy}_{\text {beam }}{ }^{-1}\right.$, corresponding to $0.19 \mu \mathrm{Jy}$ arcsec $\left.{ }^{-2}\right)$, and the solid white contours correspond to $[6,12,24,48,96] \times \sigma_{\mathrm{rms}}$. The dotted circles indicate the $R_{\mathrm{H}}$ for each model, and the smaller dashed, green circles are $r_{e}=R_{\mathrm{H}} / 2$.6. The haloes predicted from the $P_{150 \mathrm{MHz}}-M$ scaling relation (with $S_{216}=2.5 \mathrm{Jy}$, assuming $\alpha=-1.4$ ) and the upper limits are shown for $R_{\mathrm{H}}=1000 \mathrm{kpc}(14.4 \mathrm{arcmin})$ and $R_{\mathrm{H}}=500 \mathrm{kpc}(7.2 \mathrm{arcmin})$. For the top panel, Abell 3266 is featured in the lower right corner. The colour scale is the same in all panels and all panels have the same angular scale. Note the $216-\mathrm{MHz}$ robust +2.0 image with no simulated halo is shown in Figure 2. 
sources, MWA-2 data supports the lack of a central giant radio halo as noted by Bernardi et al. (2016). The MGCLS image reveal some diffuse emission connected to a central tailed galaxy (considered a 'confused tail' by Knowles et al. 2022), though the emission is largely enclosed within the $3 \sigma_{\mathrm{rms}}$ contour of the MWA-2 216-MHz image (e.g. Figure 1) around the radio sources near the cluster centre. This emission will be explored further by Riseley et al. (in prep.) but we do not consider this to be a giant radio halo.

We note the GLEAM 200-MHz image shows a high background signal around the cluster (see the bottom right panel of Figure 2), however, this is largely from residual sidelobes from the snapshot imaging process and is a feature of bright, extended sources throughout the GLEAM survey. This is particularly noticeable in the 200$\mathrm{MHz}$ wideband images. Significant source confusion occurs in the lower two MWA-2 bands near the cluster centre. It is not clear from the images at $88-$ and $118-\mathrm{MHz}$ that there is any excess diffuse emission beyond contributions from (1) discrete sources, (2) the previously noted diffuse emission, and (3) the additional ionospheric blurring that more heavily affects the $88-$ and $118-\mathrm{MHz}$ data. The less confused, higher-sensitivity $216-\mathrm{MHz}$ images do not reveal excess emission throughout the cluster that would hint towards a giant radio halo.

Based on the non-detection in the deep 216-MHz data, we investigate two possibilities: (1) a halo exists at the expected brightness based on established power-mass scaling relations (e.g. Basu 2012; Cassano et al. 2013) and is undetected in the 216-MHz ${ }^{11}$ MWA-2 data due to limited sensitivity to large angular scales, or (2) a halo, if present, is under-luminous with respect to the scaling relation. Such under-luminous haloes are being uncovered in high-sensitivity observations both in high- and low-resolution imaging. An example is the radio halo in Abell 3667: first detected in single-dish imaging from by the Parkes radio telescope (Carretti et al. 2013), followed up in low-resolution MWA-1 data (Hindson et al. 2014) and KAT-7 imaging (Riseley et al. 2015), and finally confirmed by recent MGCLS observations (de Gasperin et al. 2021) to be a giant, under-luminous radio halo ${ }^{12}$.

\subsubsection{Simulating a giant radio halo}

To simulate the giant radio halo, we estimate the expected halo brightness based on the scaling relation derived by Duchesne et al. (2021c), between cluster mass within $r_{500}{ }^{13}\left(M_{500}\right)$ and radio luminosity at $150 \mathrm{MHz}\left(P_{150 \mathrm{MHz}}\right)$ including the full literature sample of radio haloes. We note van Weeren et al. (2021) originally derived the $150-\mathrm{MHz}$ scaling relation from a sample of radio haloes detected at frequencies close to $150 \mathrm{MHz}$. This may bias the sample towards a larger fraction of ultra-steep-spectrum radio haloes (with $\alpha \lesssim-1.5$, hereinafter USSRH; Brunetti et al. 2008) which are found to be under-luminous with respect to scaling relations derived at $1.4 \mathrm{GHz}$ (Cassano et al. 2013).

For consistency with scaling relations derived by Duchesne et al. (2021c), we use the PSZ2 mass of $\sim 6.64 \times 10^{14} \mathrm{M}_{\odot}$. The expected power at $150 \mathrm{MHz}$ is $\sim 3.5 \times 10^{25} \mathrm{~W} \mathrm{~Hz}^{-1}$, corresponding to a flux

11 We choose the 216-MHz data due to the much longer integration time compared to the other bands combined with the highest nominal resolution resulting in less confusion.

12 Note a hint of emission had been reported by Johnston-Hollitt (2003), though it was unclear at the time if the emission was made up of artefacts from the nearby bright source.

13 The radius within which the mean density of the cluster is 500 times the critical density of the Universe. density of $\sim 4.2 \mathrm{Jy}$, or $\sim 2.5 \mathrm{Jy}$ at $216 \mathrm{MHz}$, assuming a spectral index of $-1.4^{14}$ (as in Duchesne et al. 2021c). The radial size of radio haloes has been found to somewhat correlate to their luminosity and host cluster properties (Feretti 2000; Cassano et al. 2007) and following the relation obtained by Cassano et al. (2007) we would expect an average radio halo radius of $\sim 450 \mathrm{kpc}$. The correlation has recently been updated by Hodgson et al. (2021a) with radio halo sizes compiled by Shakouri et al. (2016, and references therein), though they note the correlation is poor we obtain a radius of $\sim 500 \mathrm{kpc}$. As a point of comparison, we also consider a larger halo at $R_{\mathrm{H}}=1000 \mathrm{kpc}$ which serves to both ensure a suitable limit is estimated if the radius is large but also accounts for a generally smoother brightness profile. We consider a limiting case where patchy, extended emission is seen in the final image which would indicate a detection, noting that $\sigma_{\mathrm{rms}} \simeq 1.5 \mathrm{mJy} \mathrm{beam}^{-1} \simeq 0.19 \mu \mathrm{Jy} \operatorname{arcsec}^{-2}$ for the $216-\mathrm{MHz}$ robust +2.0 imaging.

Assuming $\alpha=-1.4$, frequency-dependent model radio haloes are simulated in each $216-\mathrm{MHz}$ snapshot independently following the standard circular exponential profile (e.g. Orrú et al. 2007; Murgia et al. 2009; Bonafede et al. 2009; Boxelaar et al. 2021),

$I(r)=I_{0} \exp \left(-r / r_{e}\right) \quad\left[\mathrm{Jy} \operatorname{arcsec}^{-2}\right]$,

with the peak surface brightness $I_{0}$, and the $e$-folding radius $r_{e}$ which is typically found to be $\sim R_{\mathrm{H}} / 2.6$ (Bonafede et al. 2017) for a radio halo of radius $R_{\mathrm{H}}$. We simulated haloes in two separate locations: at the cluster centre as defined by the coordinates from the MetaCatalogue of X-ray detected Clusters of galaxies (MCXC; Piffaretti et al. 2011), and in a relatively empty patch of sky near the cluster. Each location is tested independently.

To avoid the somewhat uncertain primary beam attenuation ${ }^{15}$ the model haloes are Fourier transformed into the datasets and imaged separately down to the same CLEAN threshold and with identical $u, v$ sampling and image and visibility weighting as the normal data. These snapshot-dependent model-only images are then linearly added to the normal snapshot images, allowing us to avoid the complex primary beam attenuation.

The combined model and data snapshots are then mosaicked in the usual way, though we forgo further brightness scaling and astrometry corrections. The ionospheric blurring is minimal at $216 \mathrm{MHz}$ and the flux scale deviation between snapshots is small ${ }^{16}$ and less important here as only the SNR matters for a detection. The total flux density is obtained from the input model rather than measured in the image. The $R_{\mathrm{H}}=1000 \mathrm{kpc}=14.4$ arcmin case illustrates the limitation of the MWA-2 at $216 \mathrm{MHz}$ where it is clear the recovery of the simulated halo in the stacked image is incomplete by up to a factor of $\sim 5$ due to the low SNR at this large angular scale.

The resultant models and their detection in the stacked $216-\mathrm{MHz}$ images are shown in Figure 4, with the expected flux density modelled (2.5 Jy) along with what we find to be upper limits for the quiet sky case $\left(0.8 \mathrm{Jy}\right.$ and $0.3 \mathrm{Jy}$ at $216 \mathrm{MHz}$ for radii of $R_{\mathrm{H}}=1000 \mathrm{kpc}$ and $R_{\mathrm{H}}=500 \mathrm{kpc}$, respectively) and the cluster centrecase (1.3 Jy and $0.5 \mathrm{Jy}$ ). A slightly higher limit is obtained for the cluster centredue to the residual negative sidelobes around some of the central sources which is a result of the limited deconvolution in individual snapshots.

\footnotetext{
14 For $\alpha>-1.4$, the brightness increases at $216 \mathrm{MHz}$ and the halo will become more easily detectable.

15 Particularly the difference in linear instrumental polarizations, $\mathrm{XX}$ and YY, which can vary significantly for off-zenith observations and differ from the tile-average model beam (Chokshi et al. 2021).

16 The snapshots are on average $\sim 15$ per cent brighter than expected, which is corrected during normal imaging as discussed in Section 2.1.
} 
Table 3. Derived upper limits for a giant radio halo in Abell 3266 represented by Equation 2.

\begin{tabular}{|c|c|c|c|c|}
\hline $\begin{array}{c}v \\
(\mathrm{MHz})\end{array}$ & $S_{v}^{500 \mathrm{kpc}}$ & $S_{v}^{1 \mathrm{Mpc}}$ & $\begin{array}{l}P_{v}^{500 \mathrm{kp}} \\
\left(\times 10^{2}\right.\end{array}$ & $\begin{array}{l}P_{v}^{1 \mathrm{Mpc}} \\
\left.\mathrm{Hz}^{-1}\right)\end{array}$ \\
\hline \multicolumn{5}{|c|}{ Cluster centre } \\
\hline $150^{\mathrm{a}}$ & $<800$ & $<2200$ & $<7.2$ & $<18$ \\
\hline 216 & $<500$ & $<1300$ & $<4.3$ & $<11$ \\
\hline $1400^{a}$ & $<40$ & $<90$ & $<0.3$ & $<0.8$ \\
\hline \multicolumn{5}{|c|}{ Quiet sky } \\
\hline $150^{a}$ & $<500$ & $<1300$ & $<4.3$ & $<12$ \\
\hline 216 & $<300$ & $<800$ & $<2.6$ & $<6.9$ \\
\hline $1400^{a}$ & $<20$ & $<60$ & $<0.2$ & $<0.5$ \\
\hline
\end{tabular}

Corresponding luminosities at $150 \mathrm{MHz}, 216 \mathrm{MHz}$, and $1.4 \mathrm{GHz}$ along with derived flux densities are reported in Table 3.

\subsubsection{Why do we not detect a giant radio halo?}

The derived limits place the expected $R_{\mathrm{H}}=500 \mathrm{kpc}$ radio halo to be $\sim 5$ times below the $150-\mathrm{MHz}$ scaling relations reported by Duchesne et al. (2021c), though only $\sim 2.5$ times below the scaling relations reported by van Weeren et al. (2021). If the halo were to be offset slightly from the cluster X-ray center, the limit decreases to approach the quiet sky limit, up to $\sim 8$ times below the scaling relations. Conversely, increasing the halo size to $R_{\mathrm{H}}=1000 \mathrm{kpc}$ provides a limit approximately in line with the van Weeren et al. (2021) results and up $\sim 2$ times below the Duchesne et al. (2021c) relations. Historically, such large haloes are rarely observed (see e.g. halo radii compiled by Cassano et al. 2007; Shakouri et al. 2016; Hodgson et al. 2021a) so this scenario is less likely.

Mergers are expected to trigger radio haloes in massive clusters (e.g Cassano et al. 2013), however, a number of merging cluster systems are absent of radio haloes and upper limits below the established scaling relations have been placed (e.g. Cassano et al. 2016; Bonafede et al. 2017). Additionally, some giant radio haloes have be observed to be under-luminous with respect to the $1.4 \mathrm{GHz}$ scaling relations (e.g. by a factor of $\sim 5$; Cuciti et al. 2018). Such discrepancies are usually USSRH (e.g. Brunetti et al. 2008; Cassano et al. 2013; van Weeren et al. 2021) or arise due to systematic measurementrelated problems. Incomplete $u, v$ sampling results in a loss of flux on large angular scales (e.g. Cuciti et al. 2018). Measurements with a low SNR can also under-estimate integrated flux densities which can be mitigated somewhat by fitting a model to the surface brightness profile (e.g. Bonafede et al. 2017; Boxelaar et al. 2021). In this work the flux density limits are obtained from the input model rather than measured in the resultant image avoiding this issue, and limits are derived from the 150-MHz scaling relations where USSRH are not under-luminous (e.g. Duchesne et al. 2021c). We have assumed $\alpha=-1.4$ for our simulated radio haloes, however, if we consider $\alpha=-1$ the expected halo brightness at $216 \mathrm{MHz}$ increases. Conversely, $\alpha<-4$ is required to push a $S_{150 \mathrm{MHz}}=4.2 \mathrm{Jy}$ radio halo beyond detection in the $216-\mathrm{MHz}$ image, hence, we do not consider that an ultra-steep-spectrum is responsible for the non-detection.

We instead consider the physical picture of the Abell 3266 system that may limit generation and/or observation of a giant radio halo. The observable lifetimes of haloes may be shorter than the merger processes (e.g. Donnert et al. 2013; Cassano et al. 2016) and cluster systems in a pre-merging state or at a late stage in their merger may not be observed with radio haloes as there may not be sufficient turbulence within the cluster volume to generate the radio emission (Bonafede et al. 2017). These pre-merging or late-merger systems may also be expected to host ultra-steep spectrum radio haloes $(\alpha \lesssim$ -1.5 ; Brunetti et al. 2008), though no reasonable spectral index would shift the expected brightness at $216 \mathrm{MHz}$ beyond detectability in this particular case. Some pre-merging systems have been found to host radio haloes (e.g. Ogrean et al. 2015; Duchesne et al. 2021c), and a halo is sometimes found in individual sub-clusters (Murgia et al. 2010; Botteon et al. 2018) ${ }^{17}$.

'Pre-merging', a late merger, or a weak merger? Dehghan (2014) investigated the evolution of the merger for the cluster using a Newtonian $N$-body simulation between the main two optical substructure components of the core region. The result suggested the distribution of galaxies observed represents the system $\sim 0.2-0.3$ Gyr after corepassage, predominantly involving two sub-clusters with a mass ratio of 1:2 (furthermore see Dehghan et al. 2017). Dehghan (2014) notes this is consistent with both a low-entropy tail reported by Finoguenov et al. (2006) as well as the complex radio structure in the bright WAT. Such a scenario suggests the system is not in a pre-merging state. Furthermore, the eROSITA entropy map presented by Sanders et al. (2021) support the passage of the north-east sub-cluster through the core region which has also been suggested by Sauvageot et al. (2005), after core passage $\sim 0.2 \mathrm{Gyr}$ ago.The system is therefore unlikely to be in a pre-merging state. Weak mergers may produce fainter, perhaps undetectable haloes in part due to steeper spectra (e.g. Brunetti et al. 2008; Cassano et al. 2010). A less likely scenario proposed by Sauvageot et al. (2005) suggests the the merger state is $\sim 0.8 \mathrm{Gyr}$ after core passage, however, a major merger timescale is expected to be 1-3 Gyr from simulations (e.g. Cassano et al. 2016). The optical substructure analysis presented by Dehghan et al. (2017) highlights the highly-energetic, complex, multi-component merger depositing large amounts of energy into the ICM with a mass ratio of 1:2 for the dominant central components. Such major mergers are likely a requirement for the formation of giant radio haloes (e.g. Cassano et al. 2016). Candidate shocks reported by Sanders et al. (2021) and the detection of a radio relic may further support this major merger scenario, though Sanders et al. (2021) note it is not yet clear if these are merger shocks. We therefore consider a weak merger scenario unlikely. Based on the available information on the dynamics of the cluster we cannot form a strong conclusion on the nature of this non-detection. Abell 3266 therefore joins a small collection of radio relic-hosting merging clusters without giant radio haloes.

\section{SUMMARY}

Observations of the galaxy cluster Abell 3266 with the MWA have revealed three components of diffuse, non-thermal radio emission. Two fossil sources are reported for the first time, and a previously detected radio relic towards the southern periphery of the cluster is also observed. For the relic and the northern fossil source, patchy emission is seen at $887.5 \mathrm{MHz}$ in the RACS images, though the fossil component associated with the complex WAT has no counterpart neither the RACS/SUMSS images nor the MGCLS images. The fossil sources are observed to have steep spectra across the MWA band (88$216 \mathrm{MHz}$ ), with $\alpha=-1.7 \pm 0.1$ and $\alpha=-2.4 \pm 0.4$ for the northern and western fossils, respectively. The southern radio relic appears

17 In these particular systems radio bridges have also been found between sub-clusters. 
much flatter in spectrum with $\alpha=-1.04 \pm 0.09$, hinting towards shock-driven acceleration. Additionally, we model the low-frequency spectrum of the $\sim 630 \mathrm{kpc}$ radio galaxy north of the cluster centre, finding a steep spectrum with a spectral index of $\alpha=-1.26 \pm 0.22$, suggesting significant contribution from aged plasma components.

No halo is detected above a peak surface brightness of $2 \mu \mathrm{Jy} \operatorname{arcsec}^{-2}$ at $216 \mathrm{MHz}$, providing an upper limit to the 150 MHz luminosity of $P_{150 \mathrm{MHz}}^{500 \mathrm{kpc}}=7.2 \times 10^{24} \mathrm{~W} \mathrm{~Hz}^{-1}\left(P_{150 \mathrm{MHz}}^{1 \mathrm{Mpc}}=\right.$ $18 \times 10^{24} \mathrm{~W} \mathrm{~Hz}^{-1}$ ), depending on the radius of the halo. These limits are up to $\sim 2-5$ times lower than expected from giant radio halo scaling relations at $150 \mathrm{MHz}$. Why a giant radio halo is undetected in Abell 3266 is unclear, as the cluster system appears in the exact dynamical state that would allow the formation of a giant radio halo. If a heretofore undetected radio halo is significantly under-luminous with respect to the $P_{150 \mathrm{MHz}}-M$ scaling relations, further deep observations with MeerKAT - or in the future with the upgraded MWA Phase III or SKA-Low - may uncover it.

\section{ACKNOWLEDGEMENTS}

The authors would like to thank the anonymous referee for their feedback so far that has improved the quality of this work. SWD acknowledges an Australian Government Research Training Program scholarship administered through Curtin University. CJR acknowledges financial support from the ERC Starting Grant 'DRANOEL', number 714245.

Support for the operation of the MWA is provided by the Australian Government (NCRIS), under a contract to Curtin University administered by Astronomy Australia Limited. The Australian SKA Pathfinder is part of the Australia Telescope National Facility which is managed by CSIRO. Operation of ASKAP is funded by the Australian Government with support from the National Collaborative Research Infrastructure Strategy. ASKAP uses the resources of the Pawsey Supercomputing Centre. Establishment of ASKAP, the Murchison Radio-astronomy Observatory and the Pawsey Supercomputing Centre are initiatives of the Australian Government, with support from the Government of Western Australia and the Science and Industry Endowment Fund. We acknowledge the Wajarri Yamatji people as the traditional owners of the Observatory site. This paper includes archived data obtained through the CSIRO ASKAP Science Data Archive, CASDA (https://data.csiro.au).

This research made use of a number of PYTHON packages not explicitly mentioned in the main text: APLPY (Robitaille \& Bressert 2012), ASTROPy (The Astropy Collaboration et al. 2018), MATPLOTLIB (Hunter 2007), NUMPY (van der Walt et al. 2011), sCIPY (Jones et al. 2001), and CMASHER (van der Velden 2020).

\section{DATA AVAILABILITY}

The the images underlying this article will be shared on reasonable request to the corresponding author, however, the raw data can be accessed through the MWA ASVO ${ }^{18}$ (https://asvo. mwatelescope.org/) for the MWA data. The ASKAP data is available through CASDA (https://data.csiro.au) as part of the RACS data collections (http://hdl.handle.net/102.100. $100 / 374842$ ? index=1) and the XMM-Newton data is available

18 All-Sky Virtual Observatory. through the Science Data Archive (https://www.cosmos.esa. int/web/xmm-newton/xsa).

\section{REFERENCES}

Abell G. O., Corwin Jr. H. G., Olowin R. P., 1989, ApJS, 70, 1

Arras P., Reinecke M., Westermann R., Enßin T. A., 2021, A\&A, 646, A58

Bagchi J., Durret F., Neto G. B. L., Paul S., 2006, Science, 314, 791

Bartalucci I., et al., 2017, A\&A, 608, A88

Basu K., 2012, MNRAS, 421, L112

Bernardi G., et al., 2016, MNRAS, 456, 1259

Bock D. C.-J., Large M. I., Sadler E. M., 1999, AJ, 117, 1578

Bonafede A., Giovannini G., Feretti L., Govoni F., Murgia M., 2009, A\&A, 494, 429

Bonafede A., Intema H. T., Brüggen M., Girardi M., Nonino M., Kantharia N., van Weeren R. J., Röttgering H. J. A., 2014, ApJ, 785, 1

Bonafede A., et al., 2017, MNRAS, 470, 3465

Botteon A., et al., 2018, MNRAS, 478, 885

Botteon A., et al., 2021, A\&A, 649, A37

Boxelaar J. M., van Weeren R. J., Botteon A., 2021, Astronomy and Computing, 35, 100464

Breuer J. P., Werner N., Mernier F., Mroczkowski T., Simionescu A., Clarke T. E., ZuHone J. A., Di Mascolo L., 2020, MNRAS, 495, 5014

Brunetti G., Jones T. W., 2014, International Journal of Modern Physics D, 23, 1430007

Brunetti G., et al., 2008, Nature, 455, 944

Carretti E., et al., 2013, MNRAS, 430, 1414

Cassano R., Brunetti G., Setti G., Govoni F., Dolag K., 2007, MNRAS, 378, 1565

Cassano R., Ettori S., Giacintucci S., Brunetti G., Markevitch M., Venturi T., Gitti M., 2010, ApJ, 721, L82

Cassano R., et al., 2013, ApJ, 777, 141

Cassano R., Brunetti G., Giocoli C., Ettori S., 2016, A\&A, 593, A81

Chapman J. M., Dempsey J., Miller D., Heywood I., Pritchard J., Sangster E., Whiting M., Dart M., 2017, CASDA: The CSIRO ASKAP Science Data Archive. p. 73

Chokshi A., Line J. L. B., Barry N., Ung D., Kenney D., McPhail A., Williams A., Webster R. L., 2021, MNRAS, 502, 1990

Cuciti V., Brunetti G., van Weeren R., Bonafede A., Dallacasa D., Cassano R., Venturi T., Kale R., 2018, A\&A, 609, A61

Cuciti V., et al., 2021, A\&A, 647, A51

Cypriano E. S., Sodré Laerte J., Campusano L. E., Kneib J.-P., Giovanelli R., Haynes M. P., Dale D. A., Hardy E., 2001, AJ, 121, 10

Dehghan S., 2014, PhD thesis, Victoria University of Wellington, https :// tewaharoa.victoria.ac.nz/discovery/fulldisplay?docid= alma99179242120102386\&context=L\&vid=64VUW_INST : VUWNUI\& search_scope=MyInst_and_CI\&isFrbr=true\&tab=all\&lang=en

Dehghan S., Johnston-Hollitt M., Colless M., Miller R., 2017, MNRAS, 468, 2645

Donnert J., Dolag K., Brunetti G., Cassano R., 2013, MNRAS, 429, 3564

Duchesne S. W., Johnston-Hollitt M., Zhu Z., Wayth R. B., Line J. L. B., 2020, Publ. Astron. Soc. Australia, 37, e037

Duchesne S. W., Johnston-Hollitt M., Bartalucci I., Hodgson T., Pratt G. W., 2021a, Publ. Astron. Soc. Australia, 38, e005

Duchesne S. W., Johnston-Hollitt M., Offringa A. R., Pratt G. W., Zheng Q., Dehghan S., 2021b, Publ. Astron. Soc. Australia, 38, e010

Duchesne S. W., Johnston-Hollitt M., Wilber A. G., 2021c, Publ. Astron. Soc. Australia, 38, e031

Duchesne S. W., Johnston-Hollitt M., Bartalucci I., 2021d, Publ. Astron. Soc. Australia, 38, e053

Ettori S., et al., 2019, A\&A, 621, A39

Feretti L., 2000, Observational Properties of Diffuse Halos in Clusters (arXiv: astro-ph/0006379)

Feretti L., Fusco-Femiano R., Giovannini G., Govoni F., 2001, A\&A, 373, 106

Finoguenov A., Henriksen M. J., Miniati F., Briel U. G., Jones C., 2006, ApJ, 643,790 
Fleenor M. C., Rose J. A., Christiansen W. A., Hunstead R. W., JohnstonHollitt M., Drinkwater M. J., Saunders W., 2005, AJ, 130, 957

Giacintucci S., Markevitch M., Venturi T., Clarke T. E., Cassano R., Mazzotta P., 2014, ApJ, 781, 9

Gitti M., Brunetti G., Setti G., 2002, A\&A, 386, 456

Govoni F., Feretti L., 2004, International Journal of Modern Physics D, 13, 1549

Hindson L., et al., 2014, MNRAS, 445, 330

Hodgson T., Vazza F., Johnston-Hollitt M., Duchesne S. W., McKinley B., 2021a, Stacking the Synchrotron Cosmic Web with FIGARO (arXiv:2108.13682)

Hodgson T., Bartalucci I., Johnston-Hollitt M., McKinley B., Vazza F., Wittor D., 2021b, ApJ, 909, 198

Hotan A., Whiting M., Huynh M., Moss V., 2020, ASKAP Data Products for Project AS113 (Other ASKAP pilot science including tests, TOOs or guest observations): images and visibilities. v1. CSIRO. Data Collection., http: //hdl . handle. net/102 . 100 . 100/348894? index=1

Hotan A. W., et al., 2021, Publ. Astron. Soc. Australia, 38, e009

Hunter J. D., 2007, Computing in Science and Engineering, 9, 90

Hurley-Walker N., et al., 2017, MNRAS, 464, 1146

Huynh M., Dempsey J., Whiting M. T., Ophel M., 2020, in Ballester P., Ibsen J., Solar M., Shortridge K., eds, Astronomical Society of the Pacific Conference Series Vol. 522, Astronomical Data Analysis Software and Systems XXVII. p. 263

Johnston-Hollitt M., 2003, PhD thesis, University of Adelaide, http://hdl . handle.net $/ 2440 / 21954$

Jones E., Oliphant T., Peterson P., et al., 2001, SciPy: Open source scientific tools for Python, http: //www.scipy.org/

Jones A., et al., 2021, MNRAS, 505, 4762

Knowles K., et al., 2022, A\&A, 657, A56

Liang H., Hunstead R. W., Birkinshaw M., Andreani P., 2000, ApJ, 544, 686

Mandal S., et al., 2020, A\&A, 634, A4

Martinez Aviles G., et al., 2016, A\&A, 595, A116

McConnell D., et al., 2020, Publ. Astron. Soc. Australia, 37, e048

Miller R., 2012, Bachelor's thesis, Victoria University of Wellington

Murgia M., Govoni F., Markevitch M., Feretti L., Giovannini G., Taylor G. B., Carretti E., 2009, A\&A, 499, 679

Murgia M., Govoni F., Feretti L., Giovannini G., 2010, A\&A, 509, A86

Murgia M., et al., 2011, A\&A, 526, A148

Murphy T., 1999, PhD thesis, University of Sydney, http://www . astrop. physics.usyd.edu.au/RELICS/thesis/thesis.html

Offringa A. R., Smirnov O., 2017, MNRAS, 471, 301

Offringa A. R., et al., 2014, MNRAS, 444, 606

Ogrean G. A., et al., 2015, ApJ, 812, 153

Orrú E., Murgia M., Feretti L., Govoni F., Brunetti G., Giovannini G., Girardi M., Setti G., 2007, A\&A, 467, 943

Pearce C. J. J., et al., 2017, ApJ, 845, 81

Piffaretti R., Arnaud M., Pratt G. W., Pointecouteau E., Melin J.-B., 2011, A\&A, 534, A109

Planck Collaboration et al., 2016, A\&A, 594, A27

Predehl P., et al., 2021, A\&A, 647, A1

Quici B., et al., 2021, Publ. Astron. Soc. Australia, 38, e008

Quintana H., Ramirez A., Way M. J., 1996, AJ, 112, 36

Reichardt C. L., et al., 2013, ApJ, 763, 127

Riseley C. J., 2016, PhD thesis, University of Southampton, https:// eprints.soton.ac.uk/405434/

Riseley C. J., Scaife A. M. M., Oozeer N., Magnus L., Wise M. W., 2015, MNRAS, 447, 1895

Robertson J. G., Roach G. J., 1990, MNRAS, 247, 387

Robitaille T., Bressert E., 2012, APLpy: Astronomical Plotting Library in Python, Astrophysics Source Code Library (ascl:1208.017)

Rudnick L., Cotton W., Knowles K., Kolokythas K., 2021, Galaxies, 9, 81

Sanders J. S., et al., 2021, Studying the merging cluster Abell 3266 with eROSITA (arXiv: 2106.14534)

Sauvageot J. L., Belsole E., Pratt G. W., 2005, A\&A, 444, 673

Shakouri S., Johnston-Hollitt M., Pratt G. W., 2016, MNRAS, 459, 2525

Slee O. B., Roy A. L., Murgia M., Andernach H., Ehle M., 2001, AJ, 122, 1172
The Astropy Collaboration et al., 2018, AJ, 156, 123

Tingay S. J., et al., 2013, Publ. Astron. Soc. Australia, 30, 7

Wayth R. B., et al., 2015, Publ. Astron. Soc. Australia, 32, 25

Wayth R. B., et al., 2018, Publ. Astron. Soc. Australia, 35

Wilber A., et al., 2018, MNRAS, 473, 3536

Wilber A. G., Johnston-Hollitt M., Duchesne S. W., Tasse C., Akamatsu H., Intema H., Hodgson T., 2020, Publ. Astron. Soc. Australia, 37, e040

Ye H., Gull S. F., Tan S. M., Nikolic B., 2021, MNRAS,

de Gasperin F., et al., 2021, MeerKAT view of the diffuse radio sources in Abell 3667 and their interactions with the thermal plasma (arXiv:2111.06940)

van Weeren R. J., et al., 2016, ApJ, 818, 204

van Weeren R. J., et al., 2017, Nature Astronomy, 1, 0005

van Weeren R. J., de Gasperin F., Akamatsu H., Brüggen M., Feretti L., Kang H., Stroe A., Zandanel F., 2019, Space Sci. Rev., 215, 16

van Weeren R. J., et al., 2021, A\&A, 651, A115

van der Velden E., 2020, Journal of Open Source Software, 5, 2004

van der Walt S., Colbert S. C., Varoquaux G., 2011, Computing in Science Engineering, 13, 22

This paper has been typeset from a $\mathrm{T}_{\mathrm{E}} \mathrm{X} / \mathrm{L} \mathrm{T} \mathrm{E} \mathrm{X}$ file prepared by the author. 